\title{
EP-196
}

\section{5 year study results of simultaneous pancreas resection during cytoreductive surgery and hyperthermic intraperitoneal chemotherapy}

\author{
Murat KALIN', Hanife Șeyda ÜLGÜR', Özgül DÜZGÜN*², Ömer Faruk ÖZKAN'
}

'Department of General Surgery, University of Health Sciences Istanbul Umraniye Training and Research Hospital, Istanbul, Turkey

${ }^{2}$ Department of Surgical Oncology, University of Health Sciences Istanbul Umraniye Training and Research Hospital, Istanbul, Turkey

Introduction: Aim is to present the results of application of simultaneous pancreas resection in cytoreductive surgery (CRS) and HIPEC (hyperthermic intraperitoneal chemotherapy) in patients with peritoneal carcinomatosis (PC) due to intraabdominal tumor without distant metastases.

Methods: CRS + HIPEC was performed in 264 patients with PC due to different etiologies between years 2016 to 2020 in Ümraniye Research and Training Hospital. Among these 264 patients, simultanoeus pancreas resection was performed in 43 patients. Long term mortality and morbidity of these 43 patients were evaluated on the basis of their age, sex, diagnosis, duration of operation, bleeding amount applied during HIPEC and simultaneous pancreas resection.

Results: Among these 43 patients, 20 were male, 23 were female. Average age was 59.3 (range 18 to 77). Of 43 patients with simultaneous pancreas resection 14 patients had ovarian cancer, 10 patients had colorectal cancer, 6 patients had gastric cancer, 5 patients had mesothelioma, 4 patients had sarcomatosis and 4patients had PC due to other etiologies. Average operation time was 7 hours (3 to 12), average bloodloss was $500 \mathrm{cc}$ (150 to 2,200). Among these 43 patients total pancreatectomy was performed in 3, whipple was performed in 2, subtotal pancreatectomy was performed in 24 and distal pancretectomy was performed in 14 patients. 2 patients had pancreas fistule, 1 patient had hepaticojejunostomy anastomose leakage and 3 patients died within 30 days postoperatively. Mortality rate was $6.9 \%$. Conclusions: CRS + HIPEC can be succesfully performed in patients with PC due to intrabdominal tumor without distant metastases. Pancreas resection can also be performed simultaneously with multiple organ resection in selected patients by experienced surgical teams. 